
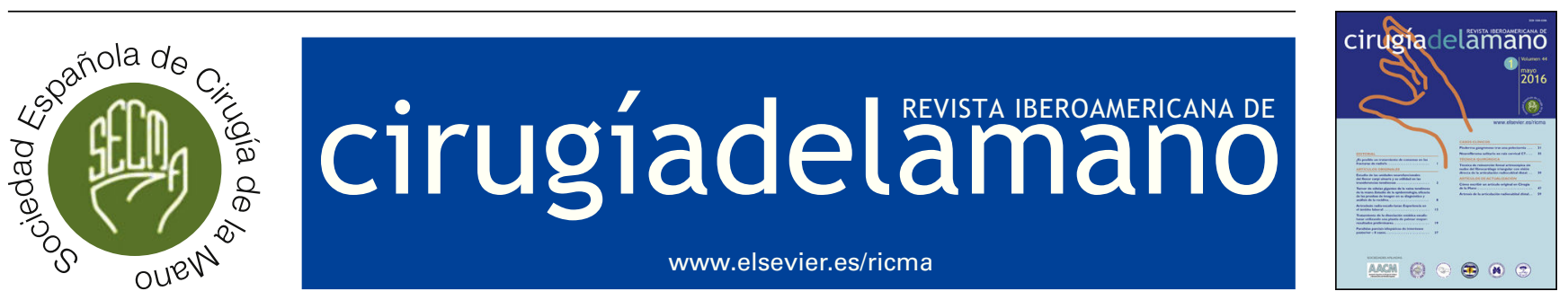

ARTÍCULO ORIGINAL

\title{
Tumores glómicos en el miembro superior. Revisión de la literatura e informe de 14 casos
}

\section{G. Celester Barreiro ${ }^{\mathrm{a}, \mathrm{b}, *}$, A. Prego Bestilleiro ${ }^{\mathrm{a}, \mathrm{b}}, \mathrm{F}$. González del Castillo ${ }^{\mathrm{a}, \mathrm{b}}$, R.M. Tobío Martel ${ }^{c}$, J. Vázquez García ${ }^{d}$ y R. Arriaza Loureda ${ }^{b}$}

\author{
a Unidad de Cirugía de la Mano, Hospital HM Modelo, A Coruña, España \\ b Instituto Médico Arriaza y Asociados, A Coruña, España \\ c Cirugía de Mano, Hospital Universitario de Caracas, Caracas, Venezuela \\ d Dermatología, Hospital HM Modelo, A Coruña, España
}

Recibido el 17 de junio de 2016; aceptado el 31 de agosto de 2016

Disponible en Internet el 19 de octubre de 2016

\section{PALABRAS CLAVE \\ Tumor glómico; \\ Glomus; \\ Glomangioma}

\begin{abstract}
Resumen
Objetivo: Revisar los resultados del tratamiento quirúrgico de los tumores glómicos del miembro superior tratados en nuestro servicio de cirugía de la mano en los últimos 15 años y comparar con la bibliografía especializada los datos de una serie de 14 tumores operados.

Material y método: Se trataron 14 tumores glómicos en 14 pacientes: 7 hombres y 7 mujeres. La media de edad fue de 50,7 años (rango 31-70).

En todos los pacientes se anotó la zona de presentación del tumor, su aspecto macroscópico con registro fotográfico, la técnica quirúrgica y el resultado final, funcional y estético.

Resultados: En todos los pacientes se dieron presentaciones únicas y todos se curaron completamente con el tratamiento quirúrgico, pero en 2 hubo recidivas y uno de ellos debió operarse hasta 5 veces para erradicar el tumor completamente.

Conclusión: El tumor glómico, por su infrecuencia, es poco conocido fuera del ámbito de la cirugía de la mano y de la dermatología, de ahí la demora en el diagnóstico que padecen buena parte de los pacientes. Los de presentación extradigital no suelen sospecharse y su diagnóstico lo aporta el estudio anatomopatológico.

El tratamiento quirúrgico, consistente en la exéresis de la masa tumoral y el legrado de la cavidad ósea si la hay, es resolutivo en la mayor parte de los casos, si bien la tasa de recidivas es muy elevada, probablemente por haberse realizado una escisión incompleta.

La complicación más frecuente es de tipo estético, por deformidades de la uña en los tumores de presentación subungueal.

( 2016 SECMA. Publicado por Elsevier España, S.L.U. Este es un artículo Open Access bajo la licencia CC BY-NC-ND (http://creativecommons.org/licenses/by-nc-nd/4.0/).
\end{abstract}

\footnotetext{
* Autor para correspondencia.

Correo electrónico: gcelester@mundo-r.com (G. Celester Barreiro).
} 


\section{KEYWORDS}

Glomus tumour; Glomus; Glomangioma
Upper limb glomus tumours. A report of 14 cases and a literature review

Abstract

Objective: To analyse the results of the surgical treatment of upper limb glomus tumours, treated in our Hand Surgery Unit, over the last 15 years, as well as to compare our data of 14 surgically treated tumours with that in the specialised bibliography.

Material and method: A total of 14 glomus tumours were treated in 14 patients ( 7 male and 7 female). The mean age was 50.7 years, with a range of 31 to 70 years.

In all patients, a record was made of the tumour presentation area, the macroscopic findings with photographs, the surgical technique, and the functional and aesthetic end results.

Results: All patients had isolated lesions and all were completely removed with the surgical treatment.

There was a recurrence in 2 cases, and 5 operations were needed in one of them to completely remove the tumour.

Conclusion: The glomus tumour, due to its low incidence, is barely known outside the hand surgery and dermatology environment. This may explain the delayed diagnosis in a high percentage of patients. The extra-digital presentation is rarely suspected and the histopathology results give us the diagnosis.

The surgical treatment, based on tumour mass excision and, if present, bone cavity curettage, resolves the vast majority of lesions. However, the recurrence rate is very high, and probably related to an incomplete excision.

The most common complication is of aesthetic nature, due to nail deformities in the subungual presentation.

(c) 2016 SECMA. Published by Elsevier España, S.L.U. This is an open access article under the CC BY-NC-ND license (http://creativecommons.org/licenses/by-nc-nd/4.0/).

\section{Introducción}

El glomus, o cuerpo glómico, es una estructura situada en la unión dermoepidérmica entre el extremo terminal de las arteriolas y el inicio de las vénulas, unidas entre sí por unos vasos anastomóticos denominados canales de Suquet Hoyer, sin interposición de capilares intermedios. Tiene la función de controlar el calibre de los pequeños vasos para regular la circulación superficial y con ello la termorregulación, por lo que está muy inervado, y de ahí la denominación de «aparato neuromioarterial» establecida por Masson en $1924^{1}$.

El tumor glómico (TG), descrito inicialmente por Wood ${ }^{2}$ en 1812, histológicamente es una hiperplasia de los elementos neuromioarteriales, o hamartoma vascular. Existen 3 tipos diferenciados (vascular, mixoide y sólido) ${ }^{1,3}$ dependiendo del componente histológico que predomine: células glómicas, estructuras vasculares o células musculares lisas, siendo el tumor sólido el más frecuente $(75 \% \text { de los casos })^{4}$.

El TG es de etiología desconocida y muy poco frecuente; representa del 1 al $5 \%$ de los tumores de la mano ${ }^{5-7}$, en la que tiene una clara tendencia a localizarse $\mathbf{7 5 \%}$ de los

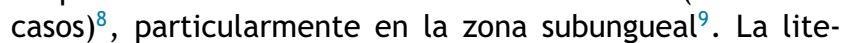
ratura refleja una mayor prevalencia en mujeres ${ }^{3,6,10}$, en las edades medias de la vida ${ }^{6,7}$, y tiene gran tendencia a recidivar.

La presentación habitual es como masa única y pequeña, que en su localización subungueal se manifiesta como un nódulo o una pápula discrómica de tonos rojo-azulados.

Debido a su infrecuencia, las series comunicadas en la literatura son de un pequeño número de casos, salvo raras excepciones, y todas muestran la dificultad del diagnóstico clínico precoz y una importante tasa de recidivas tras el tratamiento quirúrgico.
El propósito del presente trabajo fue analizar los resultados del tratamiento quirúrgico y comparar los datos obtenidos en esta serie de 14 TG con los de la literatura especializada.

\section{Material y método}

Se trata de un estudio descriptivo, en el que se analizó una serie de 14 TG del miembro superior operados desde enero de 1999 hasta enero de 2015 (15 años). Los criterios de inclusión en el estudio lo constituyeron todos los pacientes diagnosticados de TG en el miembro superior durante ese período de tiempo.

De los 14, habían consultado previamente con otros colegas 11 pacientes, 3 de los cuales venían ya correctamente diagnosticados y 2 habían recidivado tras un tratamiento previo.

Todos los casos fueron operados con gafas lupa de 20 4 aumentos; 6 pacientes fueron operados con anestesia local e isquemia con guante en la base del dedo y 8 con anestesia regional axilar y manguito de isquemia en el brazo. En los tumores extradigitales se realizó una incisión en uve sobre el punto doloroso y escisión simple de la masa tumoral. En los tumores subungueales, con una única excepción, se hizo una exéresis de la uña en todos los pacientes, una o 2 incisiones en el margen lateral del eponiquio para elevarlo, incisión longitudinal sobre la tumoración, extirpación de la misma y legrado óseo en su lugar de asiento. En un paciente se realizó abordaje lateroungueal porque el tumor había horadado una cavidad en el borde lateral de la falange (fig. 1A-C).

Cuando las pruebas de imagen mostraron invasión ósea se legró ampliamente la cavidad, sin emplear relleno en ningún 

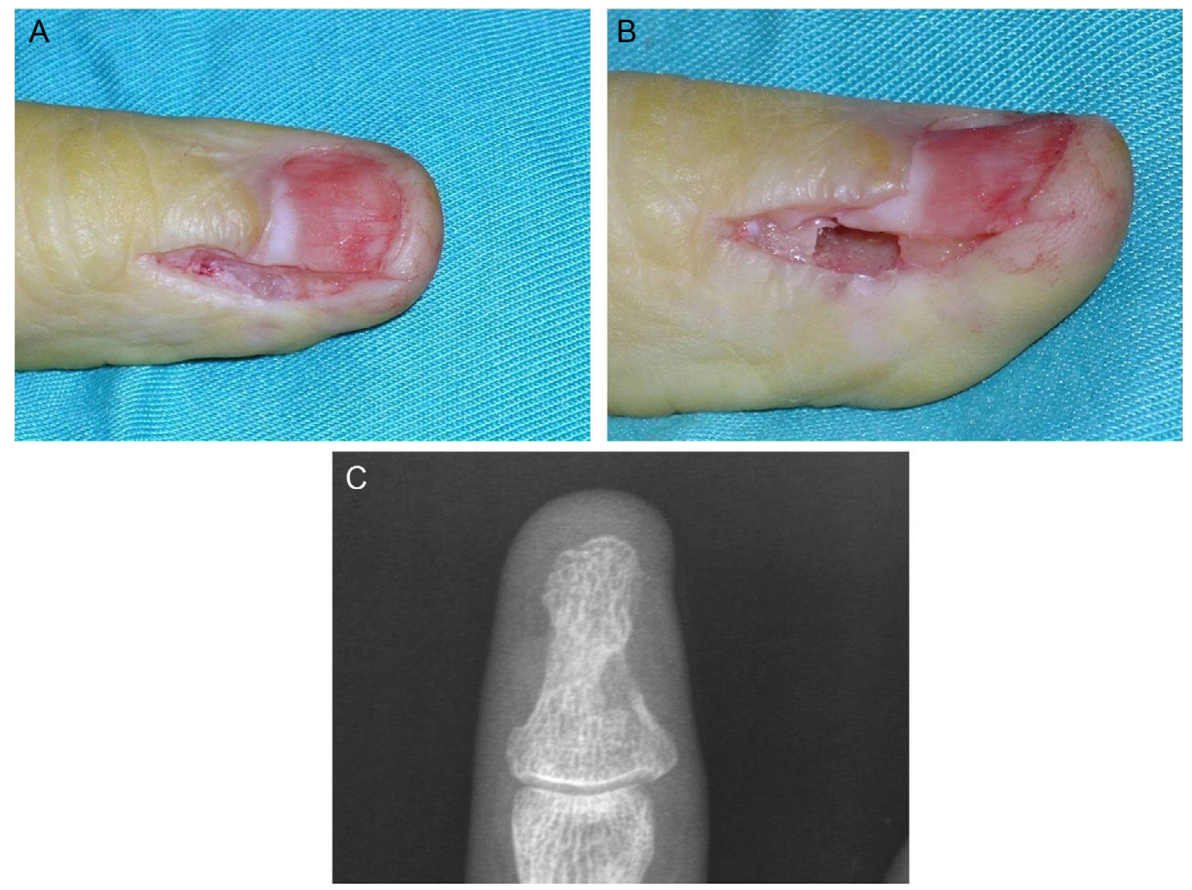

Figura 1 A-C: escisión ungueal y abordaje lateral en un paciente con invasión ósea en el borde cubital del pulgar.

caso. El lecho ungueal se suturó con hilo reabsorbible de $6 / 0$ y la piel con nylon monofilamento de 4 o $5 / 0$.

El seguimiento medio de los pacientes de la serie fue de 14 meses.

El presente estudio compara los datos demográficos de nuestra población (edad, sexo, localización) con las series más amplias encontradas en la literatura, así como el resultado estético tras el tratamiento quirúrgico y la tasa de recidivas.

\section{Resultados}

La presentación en todos los casos de esta serie fue como una masa única cuyo tamaño osciló desde $2 \mathrm{~mm}$ (una tumoración subungueal) hasta $7 \mathrm{~mm}$ (la que se asentaba en el codo), de forma redondeada, a veces como una pápula blanda, con una fina cápsula y de coloración muy variable, desde un tono pálido (fig. 2A,B) o sonrosado (fig. 3) hasta el rojo intenso (fig. 4A,B) o con matices parduzcos (fig. 5A,B).
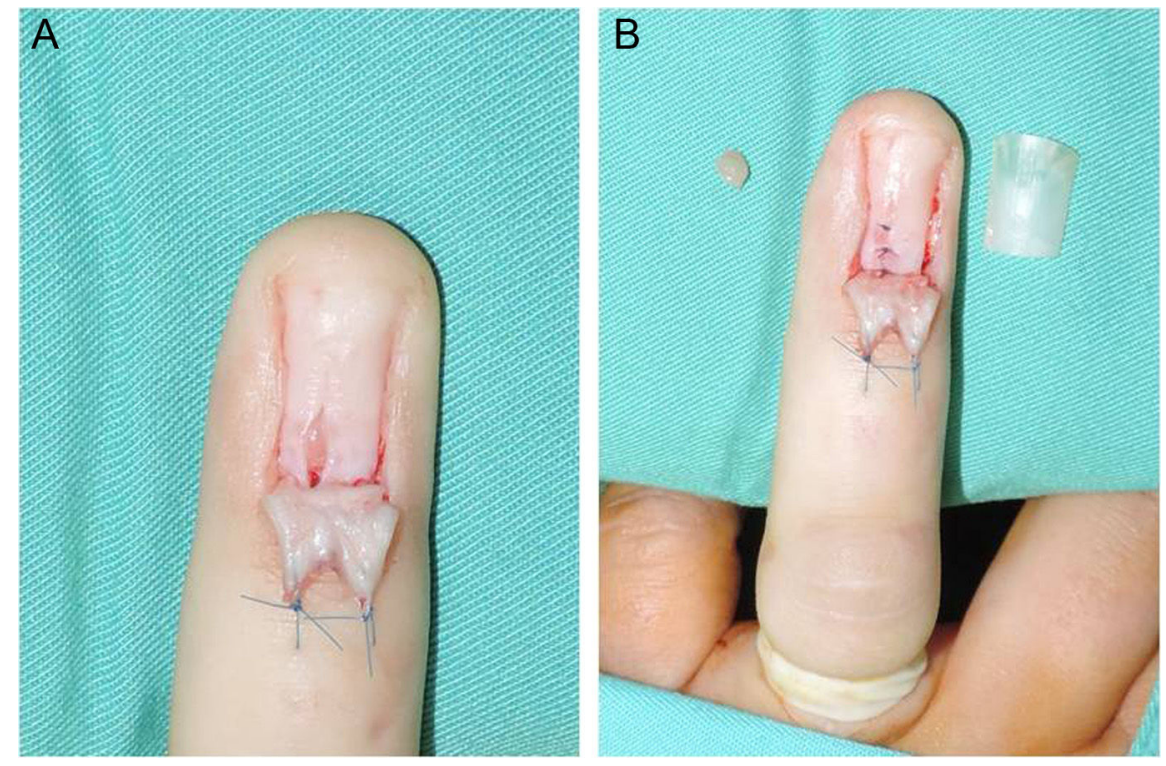

Figura 2 A y B: tumor glómico en tono pálido. 


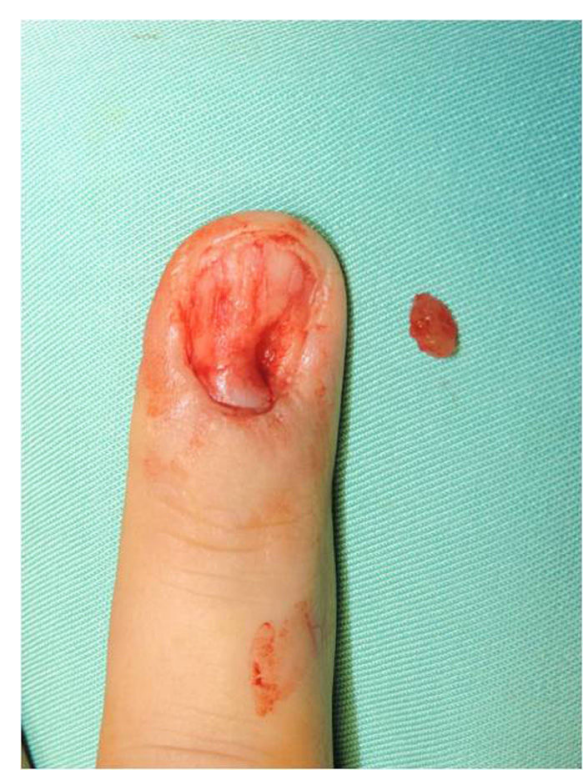

Figura 3 Tumor glómico en tono sonrosado.

Todos los pacientes se curaron sin complicaciones y se reincorporaron a sus actividades habituales sin ninguna merma funcional. Así mismo, tampoco se constató en esta serie ningún caso de distrofia ungueal significativa (fig. 6), si bien en 2 pacientes, ambas mujeres, persistió una leve cresta longitudinal bien aceptada por ellas (fig. 7). En 2 casos se empleó la propia uña para proteger la sutura del lecho, lo que previene la adhesión del eponiquio a la matriz y facilita las curas postoperatorias ${ }^{4}$ (fig. 8).

De los 14 pacientes hubo recidivas en 2 de ellos. El primer paciente con recidiva fue una mujer joven con un TG subungueal en el pulgar izquierdo, operada por el Servicio de Dermatología 2 años antes. La radiografía mostró una invasión ósea que posiblemente existiera ya en aquel momento (no se le había realizado ningún estudio de imagen) y se resolvió con el legrado de la cavidad.

El segundo paciente con recidiva fue un varón de mediana edad que precisó de 5 intervenciones hasta conseguir la curación total. Había sido operado 3 veces en otro centro. La primera intervención en nuestro servicio fue para tratar un neuroma posquirúrgico de la rama colateral cubital del pulgar de la mano derecha. La quinta y última operación, tras un seguimiento de 6 años, fue para legrar de nuevo la afectación ósea (fig. 1C).

\section{Discusión}

No se conoce la causa del TG. El examen histológico muestra la existencia de células glómicas, músculo liso y vasos sanguíneos. Hasta hace poco tiempo estaba ampliamente admitido que se trataba de un hamartoma del glomus, bien por una hipertrofia de las estructuras que lo componen, bien por una debilidad de sus vasos en posible relación con traumatismos. Pero en la actualidad existe controversia con respecto a su origen histológico. Estudios cromosómicos han identificado hasta 3 genes relacionados con el TG, localizados en el brazo largo del cromosoma $11^{11}$. En la inmunohistoquímica de las células tumorales se encontró una positividad constante de anticuerpos antivimentina, una negatividad constante del anticuerpo antifactor vIII y una positividad irregular para el anticuerpo anti-CD34 ${ }^{11}$.

CD34 es un antígeno de células precursoras del sistema hematopoyético, útil para identificar tumores vasculares y para precisar el diagnóstico de numerosos tumores de partes blandas con apariencia fusocelular ${ }^{12}$. Se trata de una proteína detectada en las células del sistema linfohematopoyético - precursoras de la serie mieloide- y se observa también en el endotelio vascular ${ }^{12}$. La negatividad de los anticuerpos antifactor vIII y anti-CD34 parece refutar el origen endotelial del TG, resultando más probable un origen de estirpe muscular.
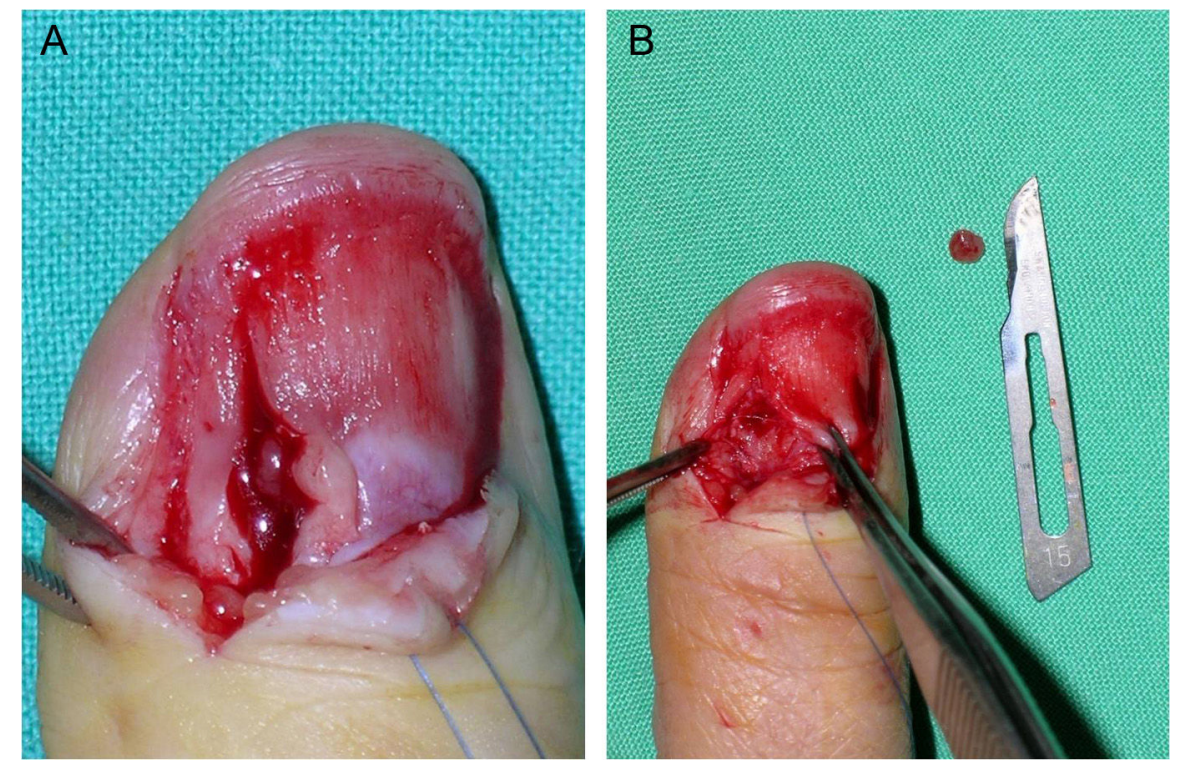

Figura 4 A y B: tumor glómico en tono rojo intenso. 


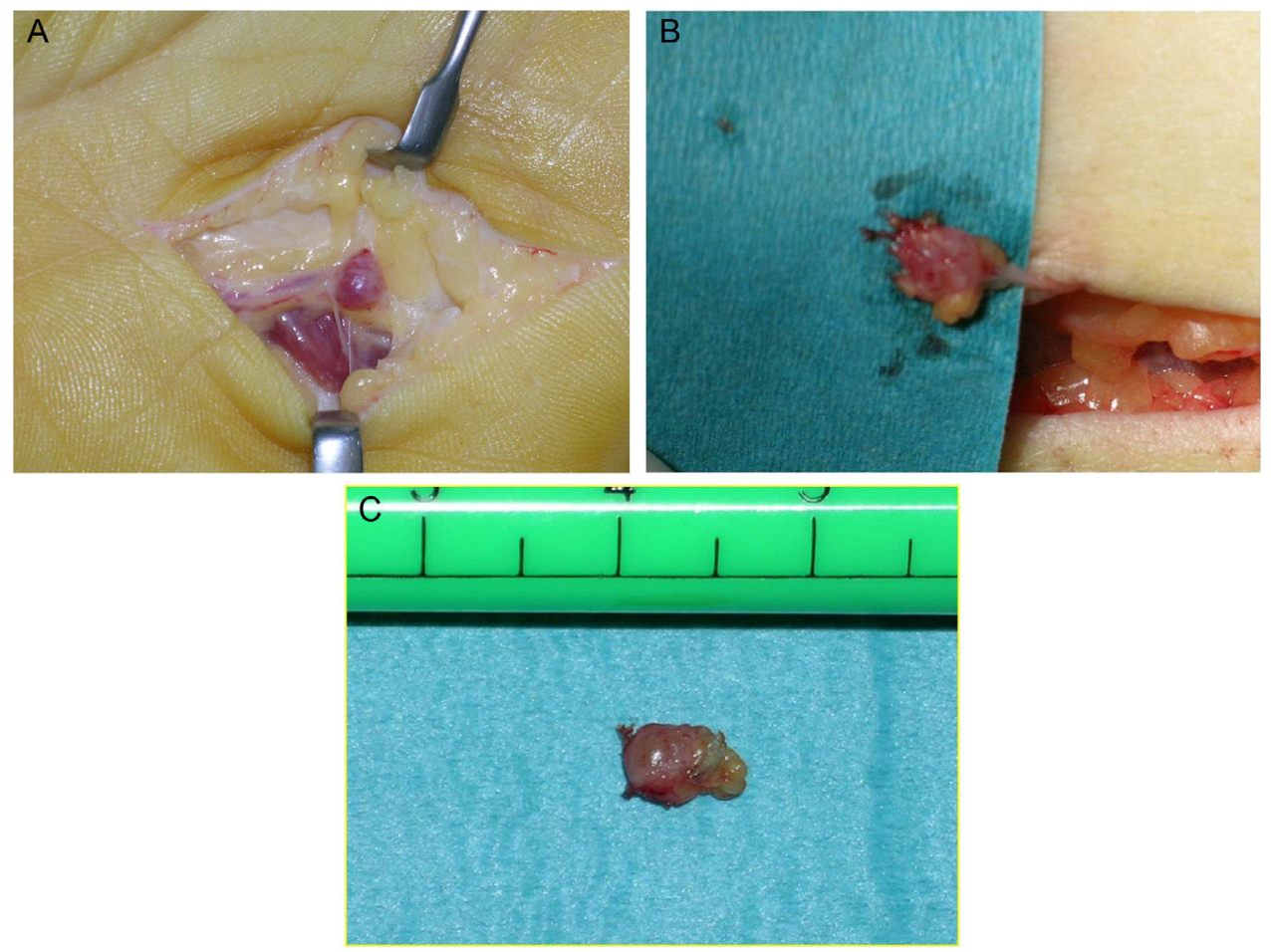

Figura 5 A-C: tumores glómicos extradigitales; A) tumor en la palma de la mano; B) tumor en la zona interna del codo; C) el tumor del codo tras su exéresis.

Los tumores del glomus representan una patología poco frecuente y poco conocida. Incluso existen publicaciones que los definen erróneamente como tumores múltiples, no dolorosos y extradigitales ${ }^{13}$.

En el diagnóstico clínico destaca como principal característica el dolor, que suele ser espontáneo, muy localizado, con exacerbaciones bruscas especialmente relacionadas con la presión o el mero tacto, incluso muy sutil. El signo de Love ${ }^{14}$ (dolor agudo a la presión puntiforme sobre la

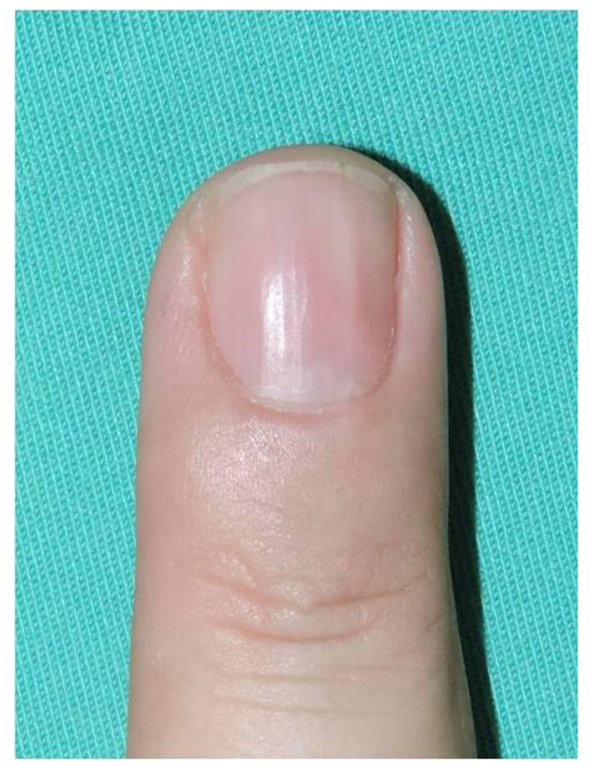

Figura 6 Aspecto estético del paciente de la figura 3. tumoración y ausencia de dolor con la misma presión en áreas adyacentes al tumor) tiene una sensibilidad del $100 \%$ y una especificidad del $0 \% 4,15$. También tienen una sensibilidad del $100 \%$ la prueba del frío (dolor al aplicar agua fría o alcohol etílico $)^{4}$ y muy alta (91-100\%) el signo de Hildreth (desaparición del dolor al realizar isquemia mediante un torniquete en el brazo ${ }^{13}$ o en la base del dedo ${ }^{15}$ ).

Otros posibles desencadenantes del dolor son los estímulos mecánicos y los cambios fisiológicos en el sexo femenino (embarazo o menstruación) ${ }^{16,17}$.

El diagnóstico suele ser tardío por diversas razones. Existen casos descritos con sintomatología previa al diagnóstico de $25^{11}$ y hasta de 40 años $^{7}$. En la literatura el diagnóstico tiene un retraso medio de 7 años desde la aparición

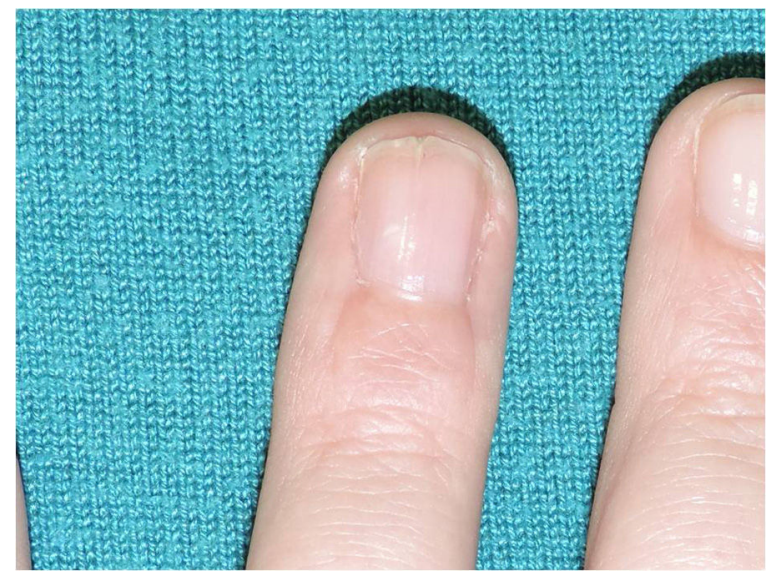

Figura 7 Aspecto estético de la paciente de las figuras 2A,B. 


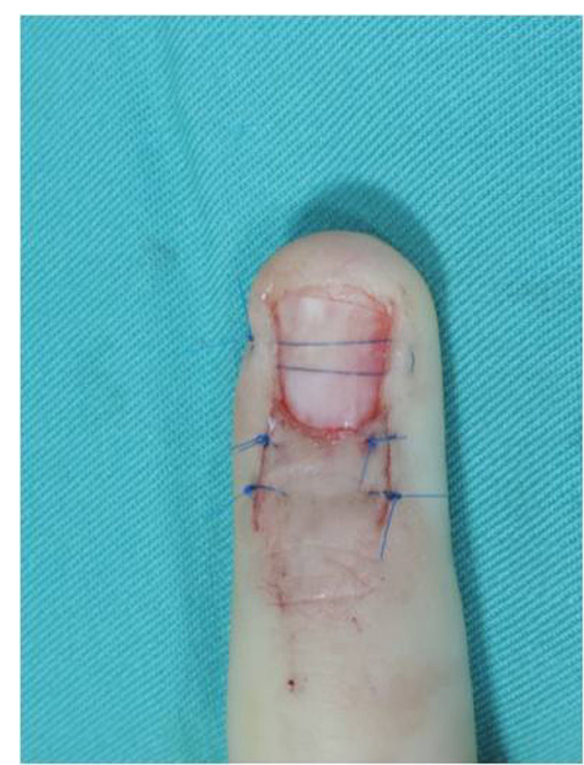

Figura 8 Reposición de la uña en la paciente de las figuras $2 A, B$ y 7 para proteger la sutura del lecho ungueal y favorecer el tratamiento postoperatorio.

de los primeros síntomas $6,10,11,17$, debido en parte a su desconocimiento y en parte a que en ocasiones cursa con una sintomatología atípica y anodina, evolucionando como una mera deformidad de la tabla ungueal ${ }^{4}$. El dolor es el síntoma más frecuente, pero no es constante; en la serie de Lin et al. ${ }^{10}$ de 75 pacientes, 15 no referían dolor.

Dentro de las pruebas de imagen es útil la radiología simple para descubrir la posible invasión intraósea, casi siempre en la falange distal de los dedos. La ecografía, con o sin Doppler, localiza bien al TG como un área hipoecoica y delimita su tamaño con precisión ${ }^{10,11,18}$, pero la prueba de elección, sobre todo si las radiografías no aportan lesiones óseas, es la RM de alta resolución, donde el TG se manifiesta como una masa bien delimitada hipointensa (oscura) en T1, y brillante en $\mathrm{T}^{8,11,19,20}$. La gammagrafía, aunque inespecífica, suele resultar positiva en todos los $\operatorname{casos}^{7}$ y la angiografía muestra una malformación vascular aproximadamente en el $50 \%$ de los $\operatorname{casos}^{7}$. Una técnica reciente en el diagnóstico del
TG es la transiluminación ${ }^{21}$, útil y económica, pero con una sensibilidad mucho menor que la $\mathrm{RM}^{15}$.

El diagnóstico preoperatorio de TG en esta serie fue sospechado solo en los casos de localización subungueal. En las presentaciones extradigitales no se habían hecho radiografías ni RM en ningún caso; el diagnóstico fue inesperado y exclusivamente anatomopatológico. A los 2 tumores situados en la palma de la mano y en el codo (fig. $5 A-C$ ) se les había realizado sendas ecografías, que no resultaron concluyentes.

El diagnóstico diferencial debe hacerse con neuroma, hemangioma subcutáneo, hiperplasia del corpúsculo de Pacini, enfermedad de Raynaud, osteoma osteoide, melanoma, nevus pigmentado y verruga vulgar, como entidades más frecuentes ${ }^{6,11,17}$, aunque también con todos los tumores dolorosos que se asienten en los dedos (exostosis subungueal, espiradenoma ecrino, leiomioma, ganglión, etc.), y aun con entidades como causalgia, artritis gotosa 0 calcinosis $^{3,4}$.

La literatura muestra un pico de TG en la $5 .^{\text {a }}$ década para los hombres y en la $4 .^{a}$ para las mujeres ${ }^{11}$. En nuestra serie la media resultó de 50,7 años (rango 31-70), más elevada que la reflejada en la literatura (tabla 1), sesgo debido a la presencia de 2 pacientes añosos (65 y 70 años).

De los 14 casos, 11 se asentaban en los dedos: 8 en la mano derecha y 3 en la izquierda: 5 en el pulgar, uno en el $2 .^{\circ}$ dedo, 2 en el $3 .^{\circ}$, uno en el $4 .^{\circ}$ y 2 en el $5 .^{\circ}$. De ellos, $9(82 \%)$ eran subungueales, uno en el pulpejo y uno en el tercio medio de la cara anterior del dedo $3 .^{\circ}$. El dedo más afectado resultó el pulgar ( 3 en la mano derecha, 2 en la izquierda). No todas las historias clínicas tenían registrado cuál era la mano dominante. De las presentaciones extradigitales, una se asentaba en el dorso de la mano sobre la base del $5 .^{\circ}$ metacarpiano, una en la palma de la mano y otra en la cara interna del codo.

En este estudio no hubo ninguna diferencia en cuanto al sexo: 7 hombres (50\%) y 7 mujeres (50\%). En un amplio estudio sobre 152 pacientes, Lee et al. ${ }^{22}$ encuentran una diferencia significativa en función del sexo en cuanto a la presentación de los TG extradigitales (33/9 a favor del sexo masculino) y digitales (20/90 de prevalencia femenina). Casi todos los autores aprecian una mayor incidencia en el sexo femenino (tabla 1).

Tabla 1 Sexo y edad media

\begin{tabular}{|c|c|c|c|c|}
\hline Autor & $\begin{array}{l}\text { Total } \\
n\end{array}$ & $\begin{array}{l}\text { Hombres } \\
\mathrm{n}(\%)\end{array}$ & $\begin{array}{l}\text { Mujeres } \\
\mathrm{n}(\%)\end{array}$ & $\begin{array}{l}\text { Edad media } \\
\text { (años) }\end{array}$ \\
\hline Marcos et al. ${ }^{6}$ & 75 & $22(29,3)$ & $53(70,7)$ & 43 \\
\hline Van Geertruyden et al. ${ }^{7}$ & 51 & $7(13,7)$ & $44(86,3)$ & 44 \\
\hline Lin et al. ${ }^{10}$ & 75 & $17(22,7)$ & $58(77,3)$ & 42 \\
\hline Abou Jaoude et al. ${ }^{11}$ & 11 & $4(36,4)$ & $7(63,6)$ & 49 \\
\hline Bhaskaranand et al. ${ }^{15}$ & 18 & $11(61,1)$ & $7(38,9)$ & 31 \\
\hline Monreal Vélez et al. ${ }^{17}$ & 11 & $2(18)$ & $9(82)$ & 37 \\
\hline Al-Qattan et al. ${ }^{20}$ & 42 & $26(61,9)$ & $16(38,1)$ & 40 \\
\hline Ekin et al. ${ }^{21}$ & 9 & $6(66,3)$ & $3(33,3)$ & 34 \\
\hline Lee et $\mathrm{al.}^{22}$ & 152 & $53(35)$ & $99(65)$ & 45 \\
\hline Lee et al. ${ }^{23}$ & 17 & $2(12)$ & $15(88)$ & 44 \\
\hline Total & 461 & $150(32,5)$ & $311(67,5)$ & 41 \\
\hline
\end{tabular}


Tabla 2 Localización de los tumores digitales

\begin{tabular}{|c|c|c|c|c|c|c|}
\hline Autor & 1. ${ }^{\text {er }}$ dedo & $2 .^{\circ}$ dedo & 3. ${ }^{\text {er }}$ dedo & $4 .^{\circ}$ dedo & $5 .^{\circ}$ dedo & Total \\
\hline Van Geertruyden et al. ${ }^{7}$ & 12 & 6 & 13 & 14 & 6 & 51 \\
\hline Abou Jaoude et al. ${ }^{11}$ & 2 & 2 & 1 & 0 & 1 & 6 \\
\hline Monreal Vélez et al. ${ }^{17}$ & 3 & 3 & 3 & 2 & 0 & 11 \\
\hline Lee et al. ${ }^{23}$ & 4 & 6 & 4 & 2 & 1 & 17 \\
\hline Serie personal & 5 & 1 & 2 & 1 & 2 & 11 \\
\hline Total & $26(27 \%)$ & $18(19 \%)$ & $23(24 \%)$ & $19(20 \%)$ & $10(10 \%)$ & 96 \\
\hline
\end{tabular}

En cuanto a la localización digital, el dedo pulgar resultó el más afectado y el meñique el que menos, como muestra la tabla 2.

La presentación en la mano suele ser como una masa única y pequeña que rara vez pasa de $1 \mathrm{~cm}$ de diámetro, aunque en el tronco o en la raíz de los miembros puede llegar a $3 \mathrm{~cm}$. En su localización subungueal se manifiestan a menudo como una pápula o discromía de tonos rojo-azulados.

Los TG de presentación múltiple, denominados glomangiomas o malformaciones glomovenosas por su apariencia angiomatosa $a^{4,24}$, son mucho más infrecuentes $(<10 \%)^{4}$, mal delimitados, no encapsulados, no suelen causar dolor, la edad de aparición es más temprana, son hereditarios, asociados a menudo con neurofibromatosis tipo । y ligados al gen de la glomulina, localizado en el cromosoma 1p21-22 24,25 .

Todos los casos de este estudio fueron de presentación única, así como en la totalidad de las series revisadas en este trabajo 4,6,7,11,15,17. El menor medía $2 \mathrm{~mm}$ y el mayor $7 \mathrm{~mm}$.

De los 14 casos de TG, 11 se asentaban en los dedos y de ellos 9 (82\%) eran subungueales. Estos datos contrastan con la serie de Van Geertruyden et al. ${ }^{7}$ en la que los 51 casos se localizaron en la falange distal.

La bibliografía no siempre recoge el porcentaje de tumores con afectación ósea y cuando lo hace muestra datos poco concluyentes. En nuestra serie había impronta ósea en 7 pacientes de los 9 con presentación subungueal. En la serie de Van Geertruyden et al. ${ }^{7}$, de los 51 casos de localización subungueal presentaron afectación ósea el $36 \%$, en la de Lin et al. ${ }^{10} 5$ de 17 casos (29\%) y en la de Monreal Vélez et al. ${ }^{17}$ solo el $9 \%$.

Los cambios de coloración son frecuentes en el TG ${ }^{6,10}$, pero el color no siempre es fácil de definir durante la intervención y puede variar en función del relleno vascular que deje la isquemia en el miembro o en la base del dedo. Los tonos más frecuentes están entre el rojo y el púrpura, aunque a menudo se presentan en color gris pálido, sonrosado o marrón.

La mayor parte de los autores revisados no comunican el color en sus observaciones, y cuando lo hacen parece predominar el tono rojo o púrpura ${ }^{10}$ (fig. 4A,B).

Se han ensayado diversos métodos de tratamiento (láser de colorante pulsado, láser de argón y CO, escleroterapia) ${ }^{4}$, pero está ampliamente admitido que el tratamiento de elección consiste en la exéresis quirúrgica, extremando la precaución de realizar una escisión completa por su alta tendencia a recidivar si quedan restos tumorales $6,7,11,20$.

Pero hay desacuerdo en cuanto a la mejor vía de abordaje quirúrgico en la localización subungueal, tanto por el mejor acceso al tumor como para evitar la distrofia ungueal posquirúrgica. Existen 2 vías clásicas. La más empleada en la literatura es la transungueal ${ }^{7,11,13,21}$ mediante una o 2 incisiones longitudinales sobre el eponiquio, levantando este para extirpar la uña y descubrir completamente el lecho ungueal; la otra es con una incisión lateroungueal elevando el lecho de la uña en bloque.

La transungueal aporta una mejor visión del tumor, pero aunque algunos autores ${ }^{4,6}$ consideran que la vía lateroungueal ofrece más garantías de evitar la distrofia ungueal, casi todos reservan esta segunda vía de acceso para los tumores localizados en las caras laterales de las falanges ${ }^{17,23}$, entre otras razones porque aunque el abordaje lateral permite visualizar y resecar el tumor, es probable que esta vía ofrezca un mayor número de recidivas. Marcos et al. ${ }^{6}$ tuvieron 14 recidivas (19\%) en 75 tumores operados por vía lateroungueal.

Con el fin de evitar la deformidad ungueal, Li et al. ${ }^{26}$ han propuesto la colocación de un injerto de grasa autógena obtenida de la zona umbilical tras la exéresis de la uña por abordaje transungueal, y colocación de una uña de silicona.

La reposición temporal de la uña como férula la hemos empleado en 3 pacientes (fig. 8); en 2 se usó la propia y en el otro caso se colocó una uña artificial empleando la pared de un frasco de colirio estéril (fig. 9A,B).

Las complicaciones posquirúrgicas más frecuentes son la recidiva y las distrofias con hendidura o despegamiento ungueal. Más raras son las parestesias secundarias y problemas de la cicatriz. De los 17 pacientes operados por Lee et al. ${ }^{23}, 9$ tuvieron deformidad ungueal, pero todos estuvieron satisfechos con el aspecto cosmético final.

El TG tiene gran tendencia a recidivar, particularidad bien reflejada en la literatura, con cifras que oscilan entre el 1 y el 50\% de los $\operatorname{casos}^{8,10,11,27}$. En nuestra serie han recidivado 2 tumores (14\%). En la serie de Lin et al. ${ }^{10}$ recidivaron el $17 \%$ de los casos, en la de Marcos et al. ${ }^{6}$ el $18,6 \%$, y en la de Al-Qattan et al. ${ }^{20}$ el $5 \%$.

Esta alta tasa de reintervenciones podría ser debida al desarrollo de un nuevo tumor ${ }^{7}$, pero en general se considera que son recidivas causadas por una escisión incompleta ${ }^{10,17,27}$ y en probable relación con el color del tumor porque en aquellos que son grisáceos o tienen una coloración similar a la cutánea, los márgenes son menos claros que cuando tienen el clásico color rojo, azul o púrpura ${ }^{10}$. Paganini et al. ${ }^{28}$ informaron de un paciente joven con 6 recidivas en un TG que se asentaba en la falange distal y solo se curó tras la resección de buena parte de la falange y un injerto córtico-esponjoso.

En ocasiones el dolor tarda en desaparecer tras la intervención, o bien reaparece la sintomatología al cabo de un tiempo, sin que ello suponga necesariamente una recidiva. En la serie de Van Geertruyden et al. ${ }^{7} 2$ pacientes 

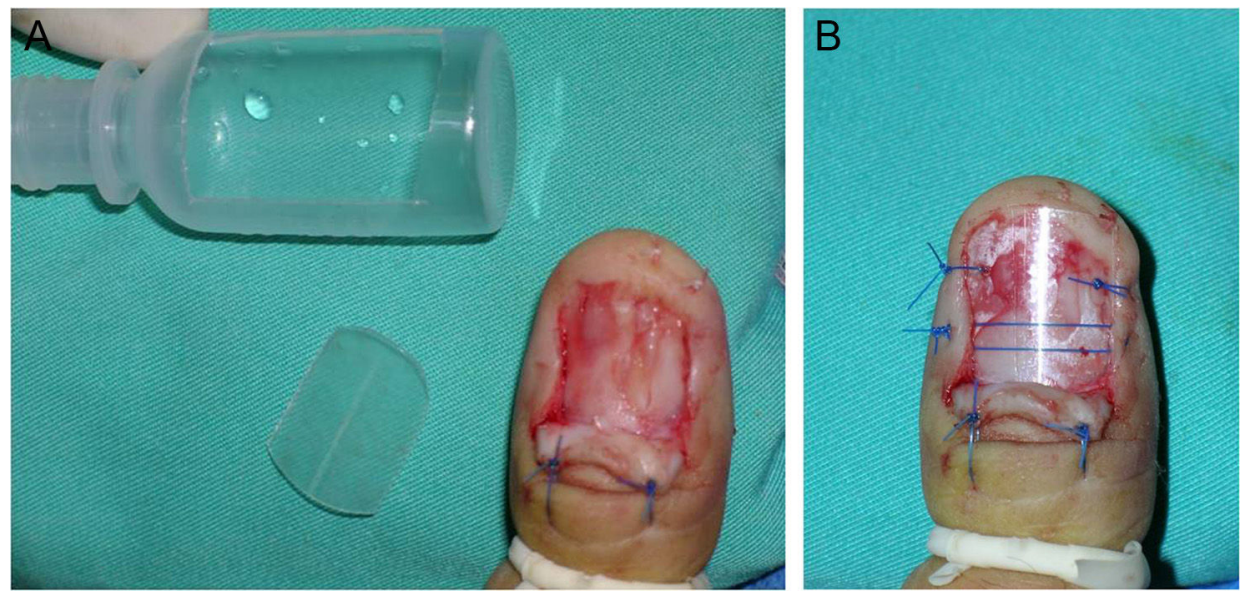

Figura 9 A y B: empleo de una uña artificial tomada de un frasco de colirio estéril.

presentaron recurrencia de síntomas a los 2 años del postoperatorio, pero ninguno precisó ser reintervenido; en la de Monreal Vélez et al. ${ }^{17}$ también hubo persistencia de síntomas en un paciente al cual no se consideró oportuno realizar una segunda intervención.

En la actualidad hay una revisión de conceptos y un cambio de criterios en cuanto al reconocimiento y clasificación de los tumores angiogénicos ${ }^{29}$, pero, en cualquier caso, la malignización de un TG a un glomangiosarcoma es muy rara. Pérez de la Fuente et al. ${ }^{30}$, en una revisión de la literatura, únicamente encontraron registro de 25 casos de glomangiosarcoma, de los cuales solo 2 se asentaban en la mano.

\section{Conclusiones}

Debe sospecharse la existencia de un TG ante un cuadro de dolor localizado (especialmente si es digital) que se exacerba con la presión puntual y el frío.

La radiografía (si hay afectación ósea), ecografía y RM localizan con precisión el tumor y delimitan el tamaño, pero el diagnóstico definitivo lo aporta el estudio histológico y las pruebas de histoquímica.

En los casos de localización extradigital el diagnóstico del TG suele ser inesperado, por la falta de sospecha; no obstante, la exéresis completa suele ser fácil y definitiva con una única intervención.

El TG tiene una alta tendencia a recidivar si no se extirpa completamente, aunque la malignización es excepcional.

\section{Responsabilidades éticas}

Protección de personas y animales. Los autores declaran que los procedimientos seguidos se conformaron a las normas éticas del comité de experimentación humana responsable y de acuerdo con la Asociación Médica Mundial y la Declaración de Helsinki.

Confidencialidad de los datos. Los autores declaran que han seguido los protocolos de su centro de trabajo sobre la publicación de datos de pacientes.
Derecho a la privacidad y consentimiento informado. Los autores han obtenido el consentimiento informado de los pacientes y/o sujetos referidos en el artículo. Este documento obra en poder del autor de correspondencia.

\section{Conflicto de intereses}

Los autores declaran no tener ningún conflicto de intereses.

\section{Bibliografía}

1. Masson P. Le glomus neuromyo-artériel des régions táctiles et ses tumours. Lyon Chir. 1924;21:256-80.

2. Wood W. On painful subcutaneous tubercle. Edinb Med J. 1812;8:283-91.

3. McDermott EM, Weiss AP. Glomus tumors. J Hand Surg Am. 2006;31:1397-400.

4. Samaniego E, Crespo A, Sanz A. Claves del diagnóstico y tratamiento del tumor glómico subungueal. Actas Dermosifiliogr. 2009;100:875-82.

5. Tuncalli D, Yilmaz AC, Terzioglu A, Aslan G. Multiple occurrences of different histologic types of the glomus tumour. J Hand Surg Am. 2005;30:161-4.

6. Marcos A, Medina J, Muratore G, Martín F, Reboso L, Foucher G. Acceso lateroungueal en los tumores glómicos periungueales. Revisión de 75 casos. Rev Iberoam Cir Mano. 2007;35:52-8.

7. Van Geertruyden J, Lorea P, Goldschmidt D, de Fontaine S, Schuind F, Kinnen L, et al. Glomus tumours of the hand. A retrospective study of 51 cases. J Hand Surg Br. 1996;21:257-60.

8. Koman LA, Ruch DS, Smith BP, Smith TL. Trastornos vasculares. Green's. Cirugía de la mano, Tomo II. Madrid: Marbán SL; 2007. p. 2312-3.

9. Sommer NZ, Brown RE. Perioniquio. Green’s. Cirugía de la mano, Tomo I. Madrid: Marbán SL; 2007. p. 414-20.

10. Lin YC, Hsiao PF, Wu YH, Sun FJ, Scher RK. Recurrent digital glomus tumor: Analysis of 75 cases. Dermatol Surg. 2010;36:1396-400.

11. Abou Jaoude JF, Roula Farah A, Sargi Z, Khairallah S, Fakih C. Glomus tumors: Report on eleven cases and a review of the literature. Chir Main. 2000;19:243-52.

12. García Tamayo J, Hernández C. Aplicación del anticuerpo CD34 en el diagnóstico diferencial de los tumores fusocelulares. VITAE. 2002; (11). Disponible en: http://caibco.ucv.ve/ caibco/vitae/VitaeOnce/Articulos/AnatomiaPatologica/ ArchivosHTML/Introduccion.htm 
13. Ofaiche J, Chauvel A, Cogrel O. Glomangiome sous-unguéal solitaire. Ann Dermatol Venereol. 2014;141:607-10.

14. Love JG. Glomus tumours: Diagnosis and treatment. Mayo Clin Proc. 1944;19:113-6.

15. Bhaskaranand K, Navadgi C. Glomus tumours of the hand. J Hand Surg Eur Vol. 2002;27:229-31.

16. Giele H. Hildreth's test is a reliable clinical sign for the diagnosis of glomus tumours. J Hand Surg Br. 2002;27:157-8.

17. Monreal Vélez JP, Rivera Vegas MJ, Valencia González J, Güemes Gordo F. Tumores glómicos de la mano: a propósito de 11 casos. Avances Traum. 1992;22:99-104.

18. Marchadier A, Cohen M, Legre R. Tumeurs glomiques sousunguéales des doigts: diagnostic échographique. Chir Main. 2006;25:16-21.

19. Salomon CG, Bednar MS, Light TR. Glomus tumor. Radiologic case study. Orthopedics. 1995;18:757-60.

20. Al-Qattan MM, Al-Namila A, Al-Thunayan A, Al-Shubi F, ElShayeb F. Magnetic resonance imaging in the diagnosis of glomus tumours of the hand. J Hand Surg Br. 2005;30:535-40.

21. Ekin A, Özkan M, Kabaklioglu T. Subungueal glomus tumours: A different approach to diagnosis and treatment. J Hand Surg Br. 1997;22:228-9.

22. Lee DW, Yang JH, Chang S, Won CH, Lee MW, Choi JH, et al. Clinical and pathological characteristics of extradigital and digital glomus tumours: A retrospective comparative study. J Eur Acad Dermatol Venereol. 2011;25:1392-7.
23. Lee IJ, Park DH, Park MC, Pae NS. Subungueal glomus tumours of the hand: Diagnosis and outcome of the transungueal approach. J Hand Surg Eur Vol. 2009;34:685-8.

24. Rodríguez Peralto JL, Alonso S, Carrillo R. Tumor glómico solitario y múltiple. En: Dermatología: Correlación clínicopatológica. España: Área Científica Menarini; 2007. p. 605-7. https://www.menarini.es/images/dermatopatologia/ Derma146.pdf

25. Redondo P. Vascular malformations (I). Concept, classification, pathogenesis and clinical features. Actas Dermosifiliog. 2007;98:141-58.

26. Li TS, Choong MY, Wu Cl, Chen HC, Chung KC. Autologous fat graft to restore nail bed contour following resection of a subungual glomus. J Hand Surg Am. 2011;36:726-8.

27. Dailiana ZH, Drapé JL, Le Viet D. A glomus tumour with four recurrences. J Hand Surg Br. 1999;24:131-2.

28. Paganini F, Aranda Romero F, Dumontier C, Le Viet D. Sexta recidiva de tumor glómico intraóseo. Rev Asoc Argent Ortop Traumatol. 2013;77:268-73.

29. Terada T, Fujimoto J, Shirakashi Y, Kamo M, Sugiura M. Malignant glomus tumor of the palm: A case report. J Cutan Pathol. 2011;38:381-4.

30. Pérez de la Fuente T, Vega C, Gutiérrez Palacios A, Sánchez Lorenzo J, González Sarasúa J. Gomangiosarcoma of the hipotenar eminence: A case report. Chir Main. 2005;24:199-202. 